\title{
Dendritic Cell-Based Therapy Using LY6E Peptide with a Putative Role Against Colorectal Cancer
}

This article was published in the following Dove Press journal:

ImmunoTargets and Therapy

\begin{abstract}
Samaneh Tokhanbigli ${ }^{1, *}$
Ali Asadirad ${ }^{2, *}$

Kaveh Baghaei'

Andrea Piccin ${ }^{3,4}$

Fatemeh Yarian ${ }^{5}$

Gilda Parsamanesh'

Seyed Mahmoud Hashemi ${ }^{6}{ }^{6}$

Hamid Asadzadeh Aghdaei (i)

Mohammad Reza Zali ${ }^{7}$

'Basic and Molecular Epidemiology of Gastrointestinal Disorders Research

Center, Research Institute for

Gastroenterology and Liver Diseases, Shahid

Beheshti University of Medical Sciences,

Tehran, Iran; ${ }^{2}$ Department of Immunology,

Faculty of Medicine, Ahvaz Jundishapur

University of Medical Sciences, Ahvaz, Iran;

${ }^{3}$ Haematology Department, Our Lady's

Children's Hospital, Dublin, Ireland;

${ }^{4}$ Department of Internal Medicine $V$,

University of Innsbruck, Innsbruck, Austria;

${ }^{5}$ Department of Biotechnology, School of

Advanced Technologies in Medicine, Shahid

Beheshti University of Medical Sciences,

Tehran, Iran; ${ }^{6}$ Department of Immunology,

School of Medicine, Shahid Beheshti

University of Medical Sciences, Tehran, Iran;

${ }^{7}$ Gastroenterology and Liver Diseases

Research Center, Research Institute for

Gastroenterology and Liver Diseases, Shahid

Beheshti University of Medical Sciences,

Tehran, Iran

*These authors contributed equally to this work
\end{abstract}

Correspondence: Kaveh Baghaei Research Institute of Gastroenterology and Liver Disease (RIGLD), Aerabi St,

Yemen St, Chamran Highway, Tehran, Iran

Tel +989123592868

Email kavehbaghai@gmail.com

Andrea Piccin

Haematology Department, Our Lady's

Children's Hospital, Dublin, Ireland

Tel +353896082831

Email apiccin@gmail.com
Introduction: Albeit early stage gastrointestinal (GI) carcinomas have a good prognosis if treated with surgery, diagnosis is often confirmed at a late stage and efficacious drugs are lacking. Recent progress in immune-based therapies has focused on dendritic cells (DCs), aiming to elicit tumor-specific responses by inducing immunological memory. Our previous microarray study indicated that a biomarker, termed lymphocyte antigen-6E (LY6E), is commonly overexpressed in two potentially lethal GI cancers: those of colon and stomach. In this study, we examined the antigenic potency of LY6E in stimulating DCs.

Methods: Following isolation, differentiation, and maturation of mononuclear cells, DCs were pulsed with LY6E peptide, a protein related to major histocompatibility complex (MHC) class I/II. Subsequently, DCs were co-cultured with mouse splenocytes to assess antigen-specific T-cell proliferation. Elucidated cytotoxic T-lymphocyte responses were assessed using subcutaneous colorectal murine tumor models.

Results: Our in vitro results suggest that DCs loaded with LY6E peptide antigen are capable of stimulating and inducing proliferation of murine T-cells. Furthermore, our in vivo results demonstrate that LY6E peptide has a substantial impact on provoking immune responses against induced colon cancer in mice.

Discussion: In conclusion, based on the overexpression of LY6E in colorectal, gastric, and pancreatic cancers, the role of this peptide should be further investigated with a goal of developing new therapies for these challenging diseases.

Keywords: dendritic cell, colorectal cancer, LY6E, dendritic cell tumor-associated antigen therapy

\section{Introduction}

Gastrointestinal (GI) cancers are a leading cause of death, with increasing incidence reported worldwide. ${ }^{1,2}$ Several therapeutic strategies have been developed, including chemotherapy, surgery, and radiation therapy as well as immune-directed therapies such as monoclonal antibodies. ${ }^{3-8}$ Antigen-presenting cells (APCs) such as dendritic cells are the primary coordinators of the innate and adaptive immune responses (humoral or antigen-specific CD8+ T-cells, respectively) that generate cytotoxic T-lymphocytes (CTLs). ${ }^{9}$

Dendritic cells play a crucial role in introducing tumor-associated antigens (TAA) to naïve T-cells in lymph nodes. The induction of specific T-cells against TAA peptides is known as the "antigen presentation" phase. Studies have shown that vaccines produced on the basis of TAA-peptides provide robust and effective CTL responses. ${ }^{10,11}$ It is imperative, therefore, to identify an appropriate tumor antigen for the design of DC tumor-based vaccines, aimed at preventing tumor 
development and inducing tumor regression. It may also be possible to design a multi-functional vaccine if a common antigen can be identified across several cancers.

Multi-purpose therapeutic anti-cancer strategies could be developed by identifying common actors that participate in metabolic pathways involved in the development of GI cancers. One of the common players that has been identified is lymphocyte antigen-6E (LY6E), a protein that is highly expressed in colorectal, gastric, and pancreatic cancers. LY6 superfamily proteins are found in leukocytes and were first observed in activated T-cells. ${ }^{12-16}$

Previous studies have identified LY6E as a common surface marker in gastric and colorectal cancers ${ }^{17}$ as well as pancreatic cancers. ${ }^{18}$ Additionally, investigation of both the Gene Expression Omnibus (GEO) database and the Georgetown Database of Cancer (G-DOC) has revealed that in comparison to adjacent tissues, LY6E had increased levels of expression in many cancers. Furthermore, worse overall survival has been directly linked to LY6E over-expression. ${ }^{15,19}$

The LY6E protein is recognized to be of particular importance in cell-cell adhesion and signal transduction through T-cell receptors and T-cell development. ${ }^{19}$ The significance of LY6E in tumor growth is postulated to involve modulation of the PTEN/PI3K/Akt/HIF-1 axis, ${ }^{20}$ a survival mechanism adopted by tumors in hypoxic environments.

Promising results have been obtained by exploring the use of antibody-drug conjugates (ADCs). In vitro and in vivo models of many cancers such as breast and ovarian, pancreatic, non-small cell lung cancer (NSCLC), and lymphoma demonstrated that ADCs targeting LY6E show promising clinical activity, with high expression of LY6E in cancer cells compared to their adjacent tissues. ${ }^{21}$

We report an in vivo study on the use of LY6E for pulsing DCs by "reverse immunology", in which the potent peptides of gene products are identified and then employed as tumor antigen against target tumors. The aim of introducing LY6E as a candidate was to design a DCTAA-based therapy to provoke CTL responses, and to investigate the effects of LY6E-pulsed DCs on an induced colorectal cancer mouse model.

\section{Materials and Methods}

\section{Animals}

Two groups of mice, each group comprising five female BALB/c aged 6-8 weeks old, were purchased from Shahid Beheshti University of Medical Sciences (Tehran, Iran). Mice were housed for at least one week prior to the onset of any experiments, to allow adaptation to environmental conditions. All experiments performed were approved by the Ethics Committee of the Research Institute for Gastroenterology and Liver Diseases (RIGID) and Shahid Beheshti Medical University (SBMU) (Code of Ethics: IR. SBMU.MSP.REC.1395.401).

\section{Peptide Prediction and Synthesis}

Immune Epitope Database and Analysis Resource (IEDB) ${ }^{22}$ was used as a prediction tool for Major Histocompatibility Complex (MHC) Class I and Class II binding sites and for the design of potential T-cell binding epitopes. Since part of the protein in its linear form binds to MHCs to provoke T-cell responses, epitope mapping was carried out for mouse MHC classes. Amongst all peptides evaluated, those with the highest ranked score (correlated with highest predicted response in provoking T-cells) were selected.

The peptide selected for this study (LY6E) with sequence of EKDHYCITLSAAAGF was designed for Mouse MHCI-II H2-IAb and was synthesized by Shine Gene Molecular Biotech (Shanghai, China), provided at $75 \%$ purity. Peptides were dissolved in Dimethyl sulfoxide (DMSO) and kept at $-80^{\circ} \mathrm{C}$ until use.

\section{Antibodies and Reagents}

Fluorochrome-conjugated (FACS) antibodies were used to characterize the immature and mature DCs and T-cell expression markers. CD40, CD14, CD11c, and CD8 (fluorescein isothiocyanate-conjugated (FITC)), CD4, CD80, CD86, and MHC II (PE-conjugated (Phycoerythrin)), and CD3 (PreCPVio700) were all obtained from eBioscience (Germany). Culture reagents and cytokines used for in vitro generation and maturation of DCs consisted of RPMI-1640 medium (Biosera, cat no: LMR1638, France) supplemented with 10\% fetal bovine serum (FBS) (Gibco, cat no 10270-106, USA), 1\% Penicillin/Streptomycin (Gibco, 151140122 USA), $50 \mu \mathrm{M}$ 2-mercaptoethanol (Sigma-Aldrich, cat no M7522, UK), 2 mM L-glutamine (Gibco, cat no 25030149, USA), Non-Essential Amino Acids (Gibco, cat no 11140050 , USA), Murine DC cytokine package $250 \mathrm{IU} / \mathrm{mL}$ recombinant murine granulocyte macrophage colony-stimulating factor (GM-CSF) and $250 \mathrm{IU} / \mathrm{mL}$ recombinant murine interleukin-4 (IL-4) (Peprotech, cat no MDC), and trypsin (Gibco, cat no: 25200056, USA).

\section{Tumor Cell Line and Culture Condition}

A murine CT26 colon carcinoma cell line $\left(\right.$ ATCC $^{\circledR}$ CRL$2638^{\mathrm{TM}}$ ) was propagated in RPMI-1640 medium containing 
$10 \%$ FBS and $1 \%$ penicillin/streptomycin according to American Type Culture Collection (ATCC) culture instructions. Cultured cells were maintained at $37^{\circ} \mathrm{C}$ and in $5 \%$ carbon dioxide (CO2). Cells with $80 \%$ confluency were harvested using trypsin.

\section{Generation and Culture of Monocyte-Derived Dendritic Cells}

Mononuclear cells were collected from femurs and tibias as previously described by Lutz et al. ${ }^{23}$ Briefly, mononuclear cell suspensions were collected in complete RPMI, containing $10 \%$ FBS and 1\% Penicillin/streptomycin, by flushing femurs and tibias of BALB/c mice. Collected cells were homogenized by pipetting. Debris was removed by passing cells through a $70 \mu \mathrm{m}$ cell strainer followed by washing of cells with phosphate-buffered saline (PBS). Cells were re-suspended in complete medium supplemented with $250 \mathrm{U} / \mathrm{mL}$ GM-CSF, 250U/mL IL-4, 1\% L-glutamine, 50 $\mu \mathrm{m}$ 2-Mercaptoethanol, $10 \% \mathrm{FBS}$, and $1 \%$ penicillin/streptomycin. The number of cells was determined and $3 \times 10^{6}$ cells were seeded in a 6-well plate. The cells were kept for three days in differentiation media, and on day $3,50 \%$ of the medium was discarded and replaced with fresh medium. After 3 and 6 days of differentiation, the phenotype of ex vivo generated DCs was microscopically examined, and immature and mature characteristics of generated DCs were analyzed by flow cytometry. On day 7 , the maturation of ex vivo generated DCs was stimulated with $100 \mathrm{ng} / \mathrm{mL}$ lipopolysaccharide (LPS) (SigmaAldrich, cat no: L4391, UK), and the culture incubation was continued overnight. Following this, non-adherent and loosely adherent cells were harvested by pipetting and centrifuged. The cells were then pulsed with $10 \mu \mathrm{g} / \mathrm{mL}$ peptide in separate groups for 3-4 hours.

\section{Flow Cytometry Analysis of Dendritic Cell and T Cell Phenotypes}

The expression of defined surface molecules was assessed by specific antibodies according to the manufacturer's protocol of eBioscience. Briefly, immature and mature DCs were harvested by pipetting and were suspended in staining buffer (PBS containing 2\% bovine serum albumin (BSA)). Cells were stained by mouse MHC Class II, CD11c, CD86, CD80, CD40, and $\mathrm{CD} 14$ at $4^{\circ} \mathrm{C}$ for 15-20 minutes in dark. Subsequently, after incubation, the cells were centrifuged in order to omit the unattached antibodies, suspended in sufficient amounts of staining buffer, and were detected by flow cytometry (BD FACScan flow cytometry system, San Jose, CA, USA).

The infiltrated T-cell lineage in tumors and extracted splenocytes were analyzed with CD3/CD4/CD8 surface markers. Dissected tumors were digested with collagenase I $(50-200 \mathrm{U} / \mathrm{mL})$ for 4 hours at $37^{\circ} \mathrm{C}$. Flushing of splenic tissue was performed using a syringe needle. The collected cells, filtrated with $70 \mu \mathrm{M}$ pore, were suspended and stained with CD3, CD4, CD8 markers for flow cytometry analysis as described above.

\section{Lymphocyte Proliferation Assay}

Division tracking dye carboxyfluorescein diacetate succinimidyl ester (CFSE) was employed to assess the stimulation property of antigen-loaded mature DCs. The splenocytes of BALB/c mice were isolated by FicollPaque PLUS (GE Healthcare cat no: 17144002, Life Sciences). These responder cells were re-suspended in PBS with a density of $1 \times 10^{6} / \mathrm{mL}$ and stained with CFSE according to the manufacturer's protocol (CCellTrace ${ }^{\mathrm{TM}}$ Cell Proliferation Kit, cat no C34570, Thermo Fisher Scientific). After 20 minutes of incubation at $37^{\circ} \mathrm{C}$ in the dark, the dye was quenched with culture medium containing $1 \%$ protein (BSA). After centrifugation, the pellet was suspended in pre-warmed culture media and applied for the proliferation assay in responder-to-stimulator ratio of 2:1, 10:1, 40:1 (splenocyte:DC). After 96 hours, cells were collected and analyzed by flow cytometry.

\section{Tumor Induction and Therapeutic Process}

Ten female BALB/c mice, aged 6-8 weeks, were divided into two groups. One group (treated) was injected with $10^{6}$ CT26 cells subcutaneously in the thigh of the animal, while the other group (untreated) acted as a control group. Seven to ten days after injection, tumor growth was assessed using an electronic calipers (Supplementary material, Figure S1), with a predetermined desired tumor size of $100-150 \mathrm{~mm}^{3}$ prior to commencing treatment. At day 10 , peptide-loaded DCs $\left(\sim 75 \times 10^{4}\right.$ cells per mouse) were injected on the contralateral side to the established tumor. Seventeen days after receiving DC loaded peptides, mice were euthanized using $100 \%$ carbon dioxide $(\mathrm{CO} 2)$ in accordance with the animal rights protocol. Following euthanasia, each of the mice were checked for absent respiration and faded eye color. The animals were then removed from the carbon dioxide chamber. A sample 
of blood was drawn from the heart and stored at $-20^{\circ} \mathrm{C}$ for further testing. Histological samples of lungs, liver, spleen, lymph nodes, and tumors were also collected and fixed in $10 \%$ paraformaldehyde and appropriately stored at $4^{\circ} \mathrm{C}$. The procedure is illustrated in Figure 1.

\section{Histopathological Examination}

After harvesting the tumors from treated and untreated mice, the dimensions of dissected tumors were noted. Tumors were fixed in 10\% paraformaldehyde and embedded in paraffin as per routine protocols. Tumor blocks were then sectioned and stained with Hematoxylin and Eosin (H\&E) according to standard techniques in order to evaluate the presence of immune cells and necrosis in tumor tissues.

\section{ELISA for Assessing Cytokine Secretion}

Serum from both treated and untreated mice was used to assess the levels of interleukin-12 (IL-12), Interferon Gamma (IFN $\gamma$ ), and interleukin-10 (IL-10) as a surrogate for elicited T-helper cell type 1 (Th1) responses, by an enzyme-linked immunosorbent assay (ELISA) kit (Bio Legend, CA, USA) according to the manufacturer's protocol.

In brief, $50 \mu \mathrm{L}$ of Matrix $\mathrm{A}$ and $50 \mu \mathrm{L}$ of Assay Buffer A were added to each well containing the standard dilutions and samples, respectively. The plate was incubated and agitated for 2 hours at room temperature. After washing, and subsequent addition of detection antibodies, the plate was incubated for an additional hour while continuously agitated. Finally, Avidin-HRP B solution, Substrate Solution D, and Stop Solution were added consecutively and the plate was analyzed both at $450 \mathrm{~nm}$ and $570 \mathrm{~nm}$.

\section{Cytotoxicity Assay}

Propidium Iodide Solution (PI) Apoptosis Detection Kit I was used to detect the cytotoxicity of extracted lymphocytes according to the manufacturer's recommendations (BD Biosciences).

CT26 cells were seeded in a 96-well plate, and after 16 hours, $10^{5}$ splenocytes were added in a 1:10 ratio. After 14-18 hours, the cytotoxicity of splenocytes was measured by propidium iodide (PI) staining using a flow cytometry technique.

\section{Statistics}

All experiments were performed three times and were presented as mean values with a standard error. Collected data were analyzed by the Graph Pad Prism software using the Student's $t$-test method. Statistical significance was recorded at a threshold $\mathrm{p}$-value of $<0.05$.

\section{Results}

\section{T Lymphocyte Proliferation Assay by Pulsed Dendritic Cells}

In the current study, the proliferation potential of DCs loaded with LY6E was investigated. We examined three different ratios of DCs and lymphocytes. In a 1:2 ratio, the proliferation of co-cultured lymphocytes in both groups of peptide-loaded DCs and mature DCs was similar. In the 1:10 ratio, proliferation of lymphocytes was significant in comparison with mature DC. However, in 1:40 group no significant proliferation was detected (Figure 2A). In the 1:10 group, proliferation of lymphocytes demonstrated significance in co-cultured lymphocytes with peptideloaded DCs in contrast with mature DCs (Figure 2B).

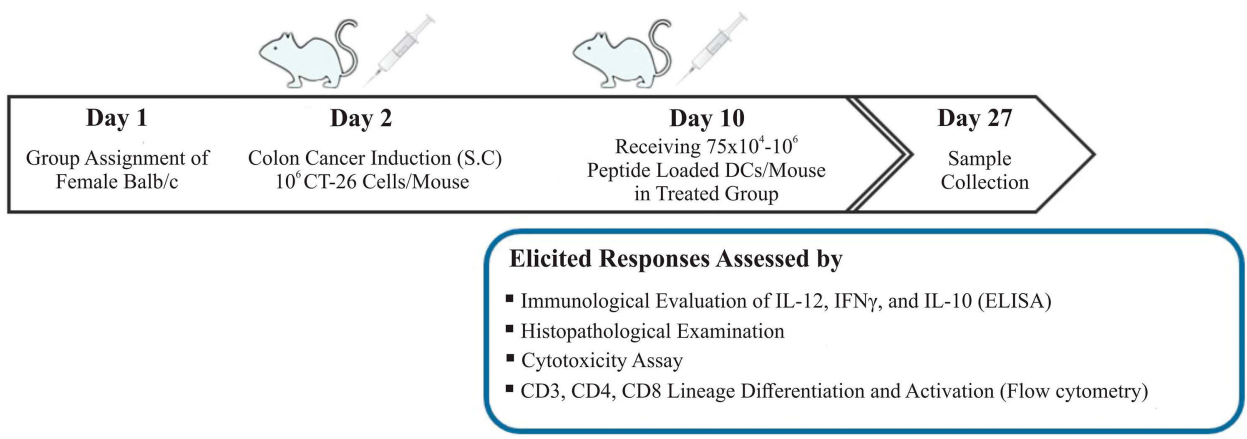

Figure I Study design and the process of inducing colorectal cancer (CRC) in BALB/c mice by CT26 cell lines subcutaneously (S.C.) and treatment process. 

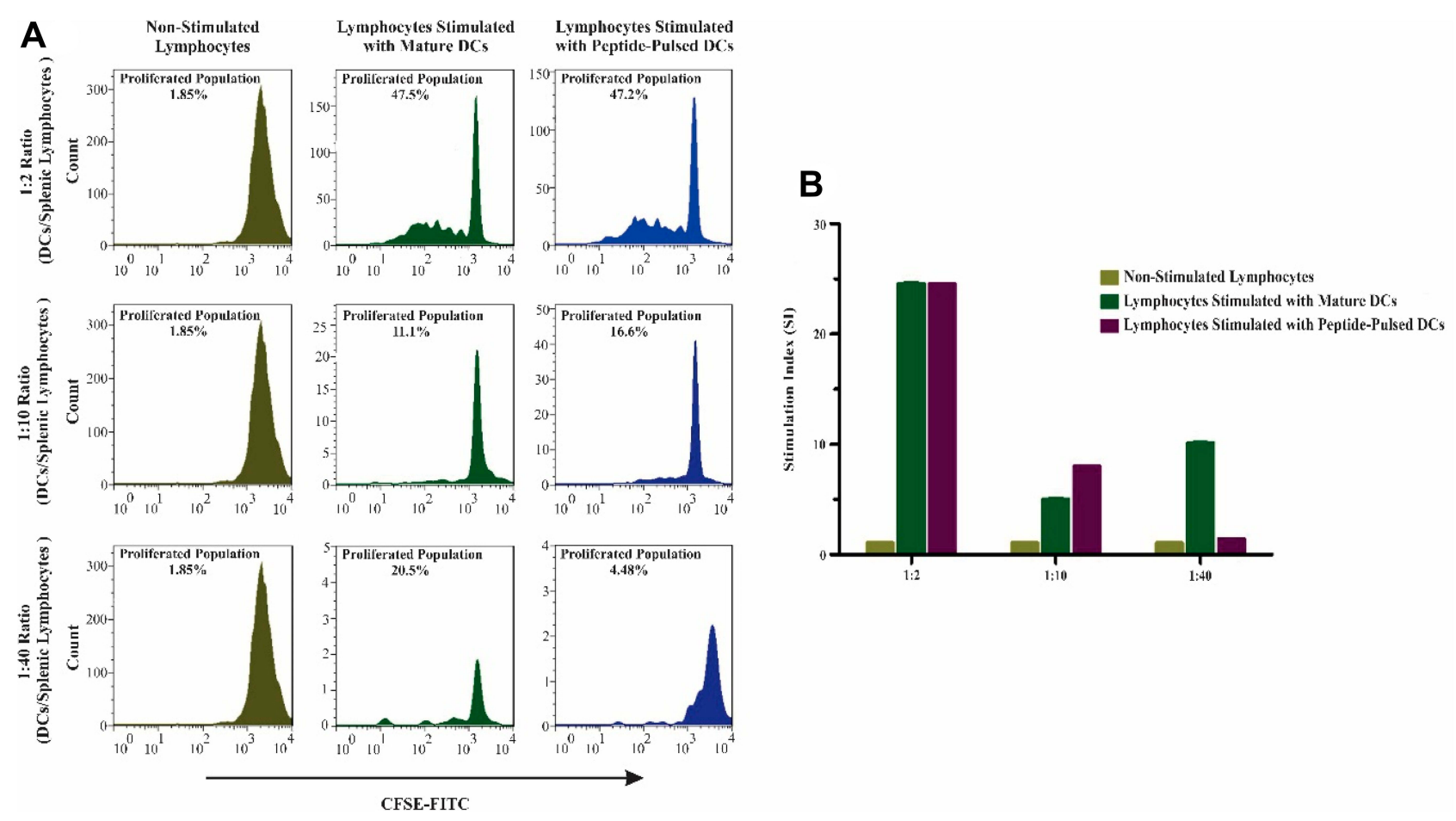

Figure 2 Proliferation and stimulation of mouse splenic lymphocytes assessed by CFSE. These results indicate that the designed peptide (LY6E) had a substantial proliferation effect on lymphocyte proliferation in contrast with unloaded mature DCs. (A) The group with I:2 mature DCs and peptide-loaded DCs stimulated splenocytes at the same levels. However, the I:40 ratio (DC/splenocyte) has shown that mature DCs provoked the proliferation of splenocytes more than loaded DCs with peptide. On the other hand, in I:I0 ratio, the results indicated that peptide-loaded DCs did efficiently proliferate splenocytes in contrast to other mature DCs and the other ratios. (B) Stimulation Index (SI) in I:I0 ratio peptide-pulsed DCs was statistically significant in comparison with the mature DCs. All quantitative data were derived from three independent experiments.

\section{Histopathology Findings of Tumor Tissues After Peptide-Loaded DC Injection}

After macroscopic examination, tumors underwent histopathological and flow cytometric analysis. In the histopathological assessment, the H\&E stained samples were analyzed for the presence of immune cells and tumor necrosis. After treatment, several morphological features of necrosis were observed. The presence of immune cells was detectable and abundant in necrotic areas, suggesting an immune response against the induced tumor (Figure 3).

\section{Immunoregulatory Effects of Dendritic Cells in Animal Model of Colorectal}

\section{Cancer}

Cytokine secretion is crucially important to elicit adequate immune and anti-tumor responses. Various T-cell types are differentiated after confrontation with signals produced by DCs. Inflammatory cells, such as Th1 cells, macrophages, and neutrophils, are determined by high levels of IL-12 and IFN- $\gamma$ and minimal changes in the expression level of IL-10. Therefore, in serum collected from mice treated with DCs pulsed with LY6E peptide compared with the untreated group, significant differences were noted in the levels of IL-12 and IFN- $\gamma$ between the groups (Figure 4).

\section{Splenic Lymphocytes Cytotoxicity Effect on CT26 Cells}

The CT26 cell line was utilized to co-culture with splenic lymphocytes and the percentage of necrotic cancerous cells was determined by using PI assay (Figure 5). Results indicated that about $43 \%$ of co-cultured cancerous cells underwent necrosis elucidating the efficient memory responses against induced cancer.

\section{T-Cell Lineage Differentiation and Activation After Receiving Peptide-Pulsed DCs}

The $\mathrm{CD}^{+}$T-cell co-receptor and $\mathrm{CD}^{+}{ }^{+}$T-cells are of pivotal importance in activating both the cytotoxic T-cell $\left(\mathrm{CD}^{+}\right.$naive T-cells). In order to assess the lineage differentiation of T-cells in mediating anti-tumor immune responses, $\mathrm{CD} 3, \mathrm{CD} 4$, and $\mathrm{CD} 8$ were quantified in 
A
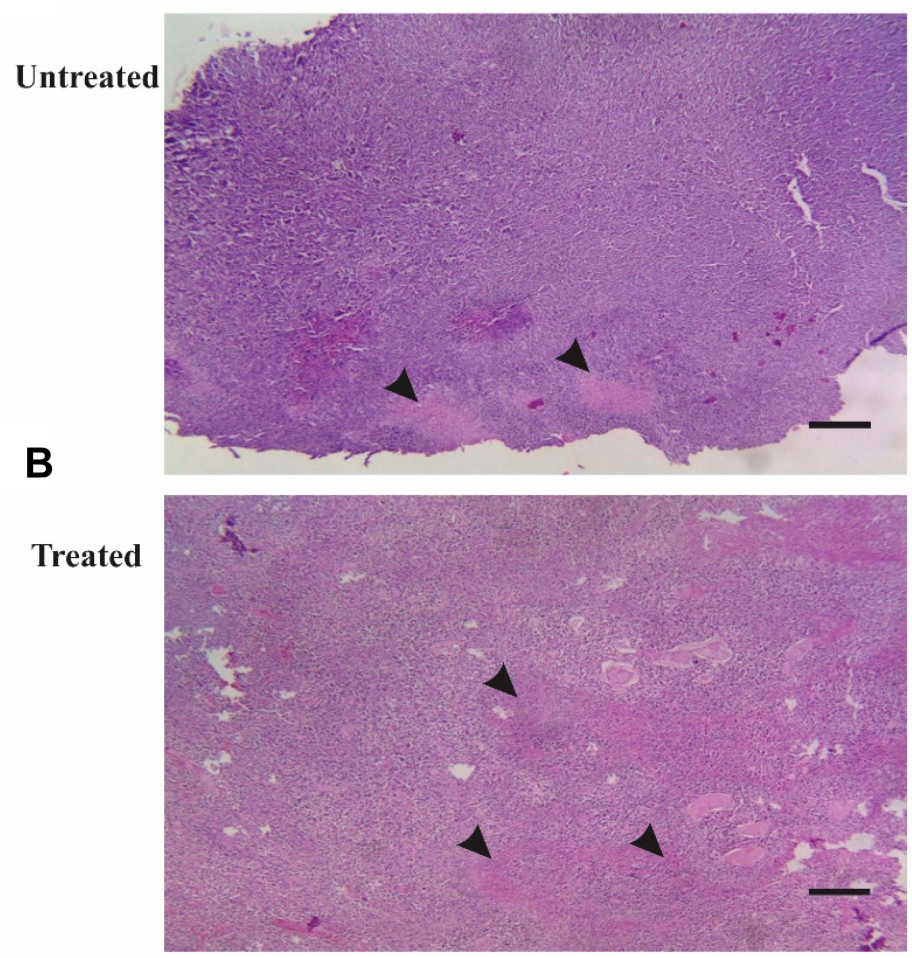

$\times 100$
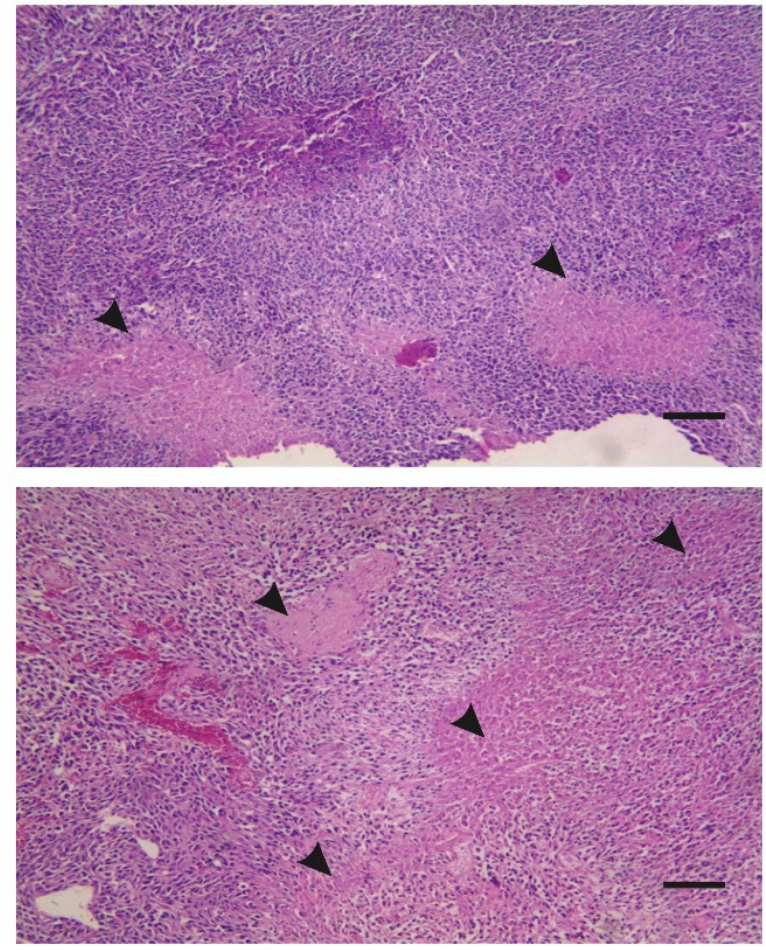

Figure 3 Histopathological outcomes from eliciting immune responses by injection of LY6E-pulsed DCs. After removing the induced tumor from untreated and treated mice, the abundance ratio of necrotic tissues demonstrates the infiltration of immune cells into induced subcutaneous tumor tissues (scale bar $5 \mu \mathrm{m}$, picture resolution $\times 40$ and $\times 100$ ). (A) In the untreated group, the necrotic environment was less than the treated group. (B) In the treated group, the infiltrated immune cells consisted of lymphocytes and neutrophils in the necrotic islands.

extracted cells of splenocytes and tumors of treated mice. Results indicate that in both tumor tissues and splenic tissues, the population of CD4 and CD8 positive cells had increased to $2.93 \%$ and $1.86 \%$, respectively. Data suggest that the LY6E-loaded DCs had the potential to provoke cytotoxic responses against induced tumor (Figure 6A). On the other hand, the $\mathrm{CD} 3+$ population showed a substantial increase from $12.4 \%$ and $7.42 \%$ in both tumor and splenocyte to $20.1 \%$ and $19.6 \%$, respectively (Figure 6B). These data are in keeping with other similar studies, indicating a significant rise in $\mathrm{CD} 3$, $\mathrm{CD} 4$, and $\mathrm{CD} 8$ populations following the addition of LY6E to DCs. ${ }^{24}$

\section{Discussion}

The key goal of immune-based therapy with dendritic cells is the identification of appropriate antigens for DC pulsing in order to provoke the anti-tumor CTLs to recognize and
IFN- $\gamma$

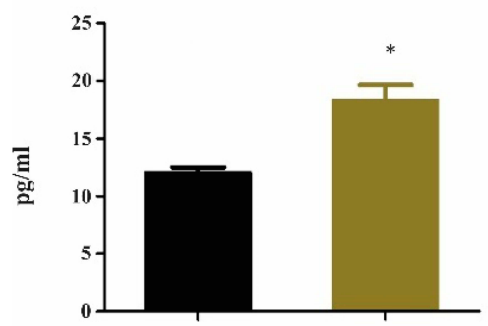

IL-12

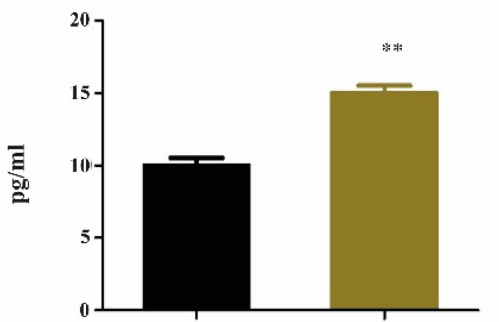

IL-10

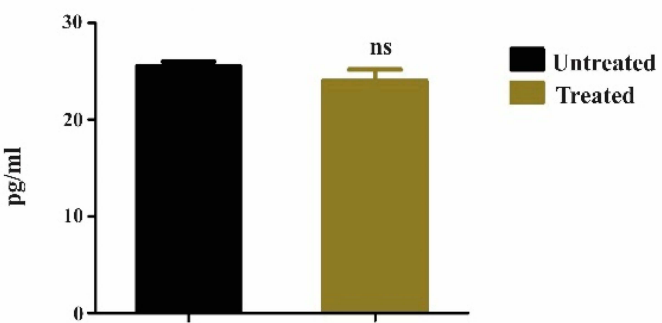

Figure 4 Immunoregulatory cytokine detection by ELISA. The release of IL-I 2 upon contact with T-cells is markedly upregulated in the treated group. Anti-tumor activity of immune cells depends on IFN- $\gamma$ produced by CD4+ T cells, showing a significant effect on tumor cells. However, as expected, levels of IL-I0 showed no statistical difference between the groups. The data are presented as mean values $\pm \mathrm{SEM}$ of experiments performed on three separate occasions. $* \mathrm{P}<0.05$, $* * \mathrm{P}<0.0 \mathrm{I}$. 


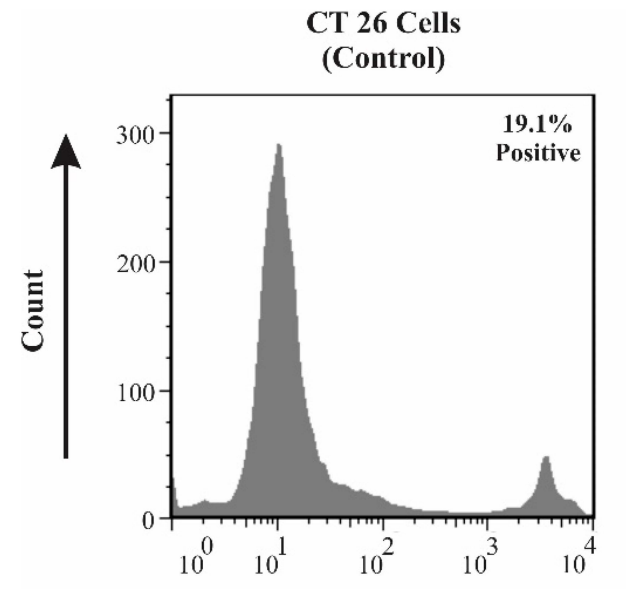

Untreated

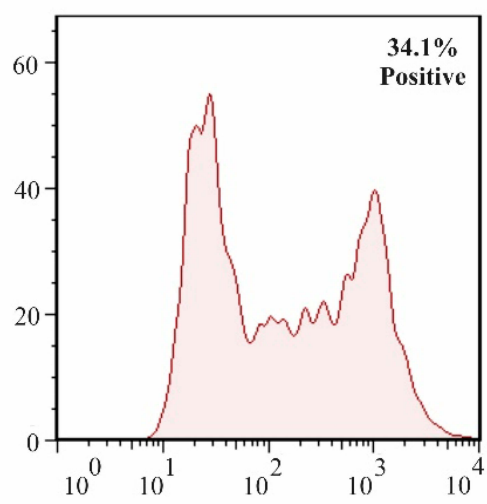

Treated

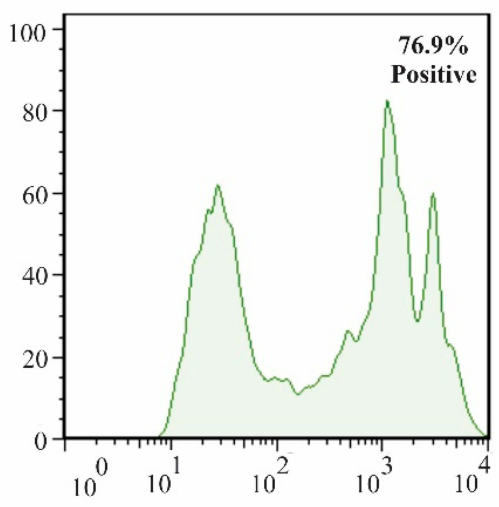

PI-PE

Figure 5 Flow cytometry-based apoptosis detection. CT26 cells were incubated with isolated splenocytes for 16 hours, and the cytotoxicity effect of activated lymphocytes on CT26 was evaluated by PI staining and flow cytometry. In this regard, in the untreated group and treated group with DC pulsed with peptide, the percentages of necrotic cells were $34.1 \%$ and $76.9 \%$, respectively.

A

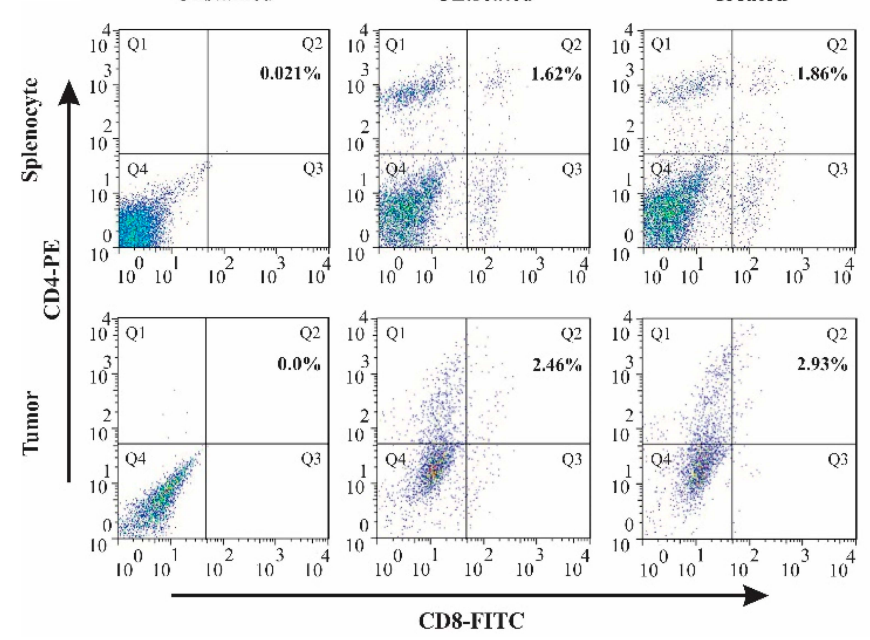

B

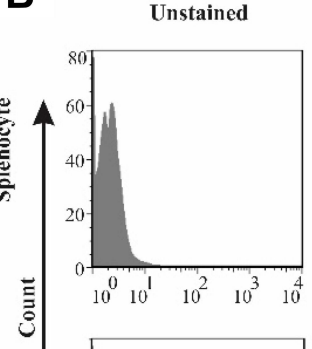

Untreated
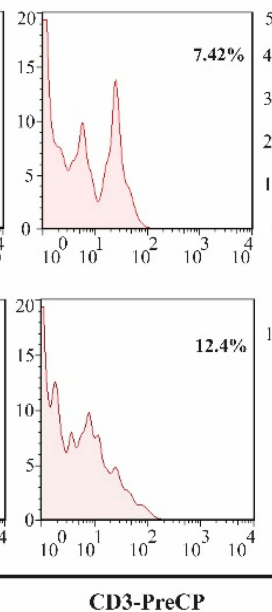

Treated
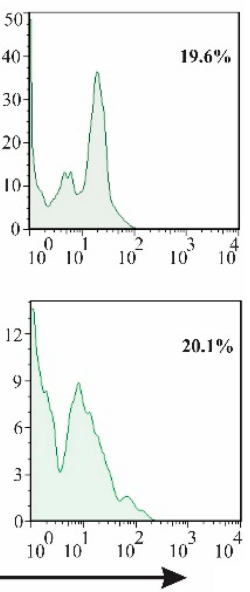

Figure 6 The stimulation of $\mathrm{CD}^{+}, \mathrm{CD}^{+}$, and $\mathrm{CD} 8^{+}$T-cells in spleen and tumor sites after $\mathrm{DC}$ therapy. The profile of T-cell population by flow cytometry. (A) The presence of CD4 and CD8 positive cells in the splenocytes of untreated and treated groups with the percentages of $1.62 \%$ and $1.86 \%$, respectively, and infiltrated population in tumor site with $2.46 \%$ in the untreated group with an increase to $2.93 \%$ in the treated group. (B) CD3 positive T-cells in the spleen of treated mice showed an increase of $7.42 \%$ to $19.6 \%$ in comparison with untreated mice. In the tumor site, the presence of CD3 positive cells was $12.4 \%$ in the untreated group, and they were elevated to $20.1 \%$ after treatment.

Abbreviations: PE, phycoerythrin; PerCP-Cy5.5, peridinin chlorophyll-cyanine.

eradicate tumor cells. Bioinformatics surveys and microarray data have demonstrated that LY6E is overexpressed in two potentially lethal GI cancers. ${ }^{17}$

The observations of our study demonstrated that DC loaded with murine LY6E peptide, which activates MHC class I and MHC class II concurrently, is able to induce anti-tumor responses in colon cancer. The importance of LY6E in many cancers has been investigated and has shown promising results both in vitro and in vivo. This comprehensive investigation was adopted on both cell lines and LY6E expressing xenograft models. The ADCs demonstrated many beneficial aspects. These drugs specifically target the cancerous cells by interfering with microtubule networks in proliferating cells, with minimal side effects on normal tissues. This occurs because the proliferation rate of normal cells is lower than the cancerous ones, with reduced cytotoxicity in normal cells. ${ }^{21}$ 
So far, few studies have been conducted using DC therapy in GI cancers. Carcinoembryonic antigen (CEA) is one of the suggested candidate antigens applied for DC therapy in colorectal cancer. Many clinical trials have been initiated based on CEA-loaded DCs with different modifications. ${ }^{10,25,26}$ The experiments in these studies were conducted on both non-transgenic and transgenic mice, and the cytotoxicity assay revealed that the CTL activity in vitro after immunization of mice with the target peptide was $20-30 \%$ with no significant difference between the two groups. ${ }^{24}$ In line with these results, our study revealed that after tumor induction and only one administration of peptide-pulsed DCs, $43 \%$ cytotoxicity effect was detected.

LY6E has been proposed to be overexpressed in ten solid tumors and is involved in the initiation of cancer progression and invasion. ${ }^{15}$ Therefore, a candidate peptide such as LY6E that targets colorectal, gastric, and pancreatic carcinoma is an appealing strategy to assist in the development of multi-purpose vaccines, based on the DCTAA peptides. Targeting multiple cancers using one peptide is an uncommon approach, and only one study has been attempted to date. MAGE Peptide was utilized to target multiple GI carcinomas (stomach, esophagus, and colon). The results of the clinical trial reported CTL responses in $4 / 8$ patients, and notably, tumor markers in seven patients were decreased. ${ }^{27}$

Furthermore, our T-lymphocyte proliferation assay results indicate that dendritic cells pulsed with MHC class I/II peptide from LY6E protein could stimulate T-cell subtypes - this finding is in accordance with previous studies. ${ }^{28}$ These encouraging results have demonstrated that proliferated lymphocytes were threefold more in pulsed DCs with LY6E than unloaded mature DCs. The capability of the LY6E peptide to provoke immune response makes it an ideal treatment alternative in the development of common tumor antigens in DC vaccines functional in multiple GI cancers.

Another example of DC therapy in GI cancer is Wilms Tumor 1 (WT1), a highly expressed oncogene implicated in a variety of malignancies, including colorectal cancer. Considering the many characteristics of WT1 in cancer progression and metastasis, a Phase I study was conducted to investigate the safety and anti-tumor responses in advanced colorectal cancer patients. After one administration of peptide, CD8+ T-cells were found to be elevated in no more than $0.6 \%$ in treated patients, and consistent with those results, the infiltrated CD $4 / 8$ positive cells ranged from $0.2 \%$ in spleen and $0.5 \%$ in tumor tissues, respectively, in our study. ${ }^{27}$ This elevation of the CD8+ population was also morphologically explicit, and pulsed LY6EDCs decreased the size of the tumors in $40 \%$ of the mice with induced colon cancer. Also, ELISA results showed a significant increase in inflammatory cytokines and activation of the immune system after DC injection. On the other hand, studies on lymphocytes isolated from the spleen revealed a reasonable increase in $\mathrm{CD}^{+} \mathrm{T}$ cells. According to reports, macrophages are predominant in the necrotic area before any treatment, and after treatment, the number of macrophages decrease, and lymphocytes and neutrophils increase in frequency. In addition, subcutaneous-induced tumors by CT26 cell lines show no metastasis to liver and lung. ${ }^{29}$ These data are in line with our current study with considerable necrotic areas in the treated group, indicating activation of the immune system against CRC tumors as a result of DC therapy with LY6E peptides.

DC pulsed with MAGE-3 peptides, ${ }^{27}$ HER-2/neuropeptides, ${ }^{30}$ and carcinoembryonic antigen RNA-pulsed $\mathrm{DCs}^{26}$ are further examples of adopting DCs in gastric cancer and colorectal cancer diseases.

The findings of our study are validated by another publication in which immunohistochemistry staining analysis of 59 of 75 human Gastric Cancer (GC) specimens proved to be LY6E positive. In this study, it was found that by silencing the LY6E expression in four different gastric cancer cell lines, cell survival, and proliferation were inhibited. As a result, cells showed cell cycle arrest in G1-S, and apoptosis was observed in AGS cell lines. On the other hand, AGS cell migration was inhibited, and increased expression of phosphatase and tensin homolog (PTEN) and E-Cadherin was detected. ${ }^{31}$ LY6E is one of the oncogenes engaged in cell adhesion, immune regulation, and drug resistance. In addition, its role has been investigated in many cancers such as lung, oesophageal, bladder, and breast cancers indicating the pivotal role of this oncogene in cancer progression. ${ }^{21,32}$

\section{Conclusions}

In conclusion, the rarity of well-characterized tumorspecific antigens in GI cancers is the main hurdle in DCbased immunotherapy. By monitoring the gene expression pathways, LY6E was discovered to be a candidate as a core actor in two potentially lethal GI malignancies: colonic and gastric cancers. In the present study, we examined the role of LY6E only in colon cancer. These findings 
suggest that LY6E should be further examined in preclinical models and should be investigated in other gastrointestinal cancers, including gastric and pancreatic cancers.

\section{Abbreviations}

GI, gastrointestinal; DCs, dendritic cells; LY6E, lymphocyte antigen-6E; MHC, major histocompatibility complex; APCs, antigen-presenting cells; CTLs, cytotoxic T-lymphocytes; TAA, tumor-associated antigens; GEO, gene expression omnibus; G-DOC, Georgetown Database of Cancer; ADCs, antibody-drug conjugates; NSCLC, non-small cell lung cancer; IEDB, Immune Epitope Database and Analysis Resource; DMSO, dimethyl sulfoxide; FACS, fluorochromeconjugated; FBS, fetal bovine serum; GM-CSF, granulocyte macrophage colony-stimulating factor; IL-4, interleukin-4; ATCC, American Type Culture Collection; CO2, carbon dioxide; PBS, phosphate-buffered saline; LPS, lipopolysaccharide; BSA, bovine serum albumin; CFSE, division tracking dye carboxyfluorescein diacetate succinimidyl ester; CRC, colorectal Cancer; S.C, subcutaneously; H\&E, hematoxylin and eosin; IL-12, interleukin-12; TNF- $\alpha$, tumor necrosis factor-alpha; IL-10, interleukin-10; Th1, T-helper cell type 1; ELISA, enzyme-linked immunosorbent assay; PI, propidium iodide solution; SI, Stimulation Index; PE, phycoerythrin; PerCP-Cy5.5, peridinin chlorophyll-cyanine; CEA, carcinoembryonic antigen; WT1, Wilms tumor 1; GC, gastric cancer; PTEN, phosphatase and tensin homolog.

\section{Ethical Approval}

Prior to conducting the research, project protocols were reviewed and approved by the ethics committees of the Shahid Beheshti University of Medical Sciences (SBMU) and the Research Institute for Gastroenterology and Liver Diseases (RIGLD). The approved code of ethics (Code of Ethics: IR.SBMU.MSP.REC.1395.401) was in accordance with the ethical standards of these institutes.

\section{Acknowledgments}

We thank Dr. Stefan Nierkens for assistance with comments that significantly improved the manuscript. The represented study was funded and supported by The Research Institute for Gastroenterology and Liver Diseases (RIGLD), Shahid Beheshti University of Medical Sciences (Tehran, Iran)

\section{Disclosure}

We declare that we have no conflicts of interest.

\section{References}

1. Pourhoseingholi MA, Vahedi M, Baghestani AR. Burden of gastrointestinal cancer in Asia; an overview. Gastroenterol Hepatol Bed Bench. 2015;8(1):19.

2. Herszenyi L, Tulassay Z. Epidemiology of gastrointestinal and liver tumors. Eur Rev Med Pharmacol Sci. 2010;14(4):249-258.

3. Ilic M, Ilic I. Epidemiology of pancreatic cancer. World J Gastroenterol. 2016;22(44):9694. doi:10.3748/wjg.v22.i44.9694

4. Wong MCS, Jiang JY, Liang M, Fang Y, Yeung MS, Sung JJY. Global temporal patterns of pancreatic cancer and association with socioeconomic development. Sci Rep. 2017;7(1):1-9. doi:10.1038/ s41598-016-0028-x

5. Favoriti P, Carbone G, Greco M, Pirozzi F, Pirozzi REM, Corcione F. Worldwide burden of colorectal cancer: a review. Updates Surg. 2016;68(1):7-11. doi:10.1007/s13304-016-0359-y

6. Douaiher J, Ravipati A, Grams B, Chowdhury S, Alatise O, Are C. Colorectal cancer-global burden, trends, and geographical variations. J Surg Oncol. 2017;115(5):619-630. doi:10.1002/jso.24578

7. Tewari M, Sahai S, Mishra RR, Shukla SK, Shukla HS. Dendritic cell therapy in advanced gastric cancer: a promising new hope? Surg Oncol. 2012;21(3):164-171. doi:10.1016/j.suronc.2012.03.003

8. Ajani JA, Lee J, Sano T, Janjigian YY, Fan D, Song S. Gastric adenocarcinoma. Nat Rev Dis Primers. 2017;3(1):1-19.

9. Langenkamp A, Messi M, Lanzavecchia A, Sallusto F. Kinetics of dendritic cell activation: impact on priming of $\mathrm{T} \mathrm{H} \mathrm{1,} \mathrm{T} \mathrm{H} 2$ and nonpolarized T cells. Nat Immunol. 2000;1(4):311. doi:10.1038/79758

10. Kantor J, Irvine K, Abrams S, Kaufman H, Dipietro J, Schlom J. Antitumor activity and immune responses induced by a recombinant carcinoembryonic antigen-vaccinia virus vaccine. J Natl Cancer Inst. 1992;84(14):1084-1091. doi:10.1093/jnci/84.14.1084

11. Collignon A, Perles-Barbacaru AT, Robert S, et al. A pancreatic tumor-specific biomarker characterized in humans and mice as an immunogenic onco-glycoprotein is efficient in dendritic cell vaccination. Oncotarget. 2015;6(27):23462. doi:10.18632/oncotarget. 4359

12. Vigneron N, Stroobant V, van den Eynde BJ, van der Bruggen P. Database of $\mathrm{T}$ cell-defined human tumor antigens: the 2013 update. Cancer Immun. 2013;13(3):1-6.

13. Luo L, McGarvey P, Madhavan S, Kumar R, Gusev Y, Upadhyay G. Distinct lymphocyte antigens 6 (Ly6) family members Ly6D, Ly6E, Ly6K and Ly6H drive tumorigenesis and clinical outcome. Oncotarget. 2016;7(10):11165-11193.

14. McKenzie IFC, Cherry M, Snell GD. Ly-6.2: a new lymphocyte specificity of peripheral T-cells. Immunogenetics. 1977;5(1):25-32. doi:10.1007/BF01570460

15. Kong HK, Park JH. Characterization and function of human Ly-6/ uPAR molecules. BMB Rep. 2012;45(11):595. doi:10.5483/ BMBRep.2012.45.11.210

16. Lee PY, Wang J, Parisini E, Dascher CC, Nigrovic PA. Ly6 family proteins in neutrophil biology. J Leukoc Biol. 2013;94(4):585-594. doi:10.1189/jlb.0113014

17. Baghaei K, Hosseinkhan N, Aghdaei HA, Zali MR. Investigation of a common gene expression signature in gastrointestinal cancers using systems biology approaches. Mol Biosyst. 2017;13(11):2277-2288. doi:10.1039/C7MB00450H

18. Alldinger I, Dittert D, Peiper M, et al. Gene expression analysis of pancreatic cell lines reveals genes overexpressed in pancreatic cancer. Pancreatology. 2005;5(4-5):370-379. doi:10.1159/000086537

19. Classon BJ, Boyd RL. Thymic-shared antigen-1 (TSA-1) A lymphostromal cell membrane Ly-6 superfamily molecule with a putative role in cellular adhesion. Clin Dev Immunol. 1998;6(1-2):149-156. doi:10.1155/1998/53157

20. Yeom CJ, Zeng L, Goto Y, et al. LY6E: a conductor of malignant tumor growth through modulation of the PTEN/PI3K/Akt/HIF-1 axis. Oncotarget. 2016;7(40):65387. 
21. Asundi J, Crocker L, Tremayne J, et al. An antibody-drug conjugate directed against lymphocyte antigen 6 complex, locus E (LY6E) provides robust tumor killing in a wide range of solid tumor malignancies. Clin Cancer Res. 2015;21(14):3252-3262. doi:10.1158/ 1078-0432.CCR-15-0156

22. Vita R, Overton JA, Greenbaum JA, et al. The immune epitope database (IEDB) 3.0. Nucleic Acids Res. 2015;43(D1):D405-12. doi:10.1093/nar/gku938

23. Kizilay Mancini O, Shum-Tim D, Stochaj U, Correa JA, Colmegna I. Age, atherosclerosis and type 2 diabetes reduce human mesenchymal stromal cell-mediated T-cell suppression. Stem Cell Res Ther. 2015;6 (1):6-10. doi:10.1186/s13287-015-0127-9

24. Bae MY, Cho NH, Seong SY. Protective anti-tumour immune responses by murine dendritic cells pulsed with recombinant Tat-carcinoembryonic antigen derived from Escherichia coli. Clin Exp Immunol. 2009;157 (1):128-138. doi:10.1111/j.1365-2249.2009.03943.x

25. Liu KJ, Wang CC, Chen LT, et al. Generation of carcinoembryonic antigen (CEA)-specific T-cell responses in HLA-A*0201 and HLAA*2402 late-stage colorectal cancer patients after vaccination with dendritic cells loaded with CEA peptides. Clin Cancer Res. 2004;10 (8):2645-2651. doi:10.1158/1078-0432.CCR-03-0430

26. Lesterhuis WJ, de Vries IJM, Schreibelt G, et al. Immunogenicity of dendritic cells pulsed with CEA peptide or transfected with CEA mRNA for vaccination of colorectal cancer patients. Anticancer Res. 2010;30(12):5091-5097.
27. Sadanaga N, Nagashima H, Mashino K, et al. Dendritic cell vaccination with MAGE peptide is a novel therapeutic approach for gastrointestinal carcinomas 1. Clin Cancer Res. 2001;7:2277-2284.

28. Lutz MB, Kukutsch N, Ogilvie ALJ, et al. An advanced culture method for generating large quantities of highly pure dendritic cells from mouse bone marrow. J Immunol Methods. 1999;223(1):77-92. doi:10.1016/S0022-1759(98)00204-X

29. Westwood JA, Ellis S, Danne J, et al. An ultrastructural investigation of tumors undergoing regression mediated by immunotherapy. Oncotarget. 2017;8(70):115215. doi:10.18632/oncotarget.23215

30. Kono K, Takahashi A, Sugai H, et al. Dendritic cells pulsed with HER-2/neu-derived peptides can induce specific T-cell responses in patients with gastric cancer. Clin Cancer Res. 2002;8(11):3394-3400.

31. Lv Y, Song Y, Ni C, et al. Overexpression of lymphocyte antigen 6 complex, locus $\mathrm{E}$ in gastric cancer promotes cancer cell growth and metastasis. Cell Physiol Biochem. 2018;45(3):1219-1229. doi:10.11 $59 / 000487453$

32. AlHossiny M, Luo L, Frazier WR, et al. Ly6E/K signaling to TGF $\beta$ promotes breast cancer progression, immune escape, and drug resistance. Cancer Res. 2016;76(11):3376-3386. doi:10.1158/0008-5472. CAN-15-2654
ImmunoTargets and Therapy

\section{Publish your work in this journal}

ImmunoTargets and Therapy is an international, peer-reviewed open access journal focusing on the immunological basis of diseases, potential targets for immune based therapy and treatment protocols employed to improve patient management. Basic immunology and physiology of the immune system in health, and disease will be also covered. In addition, the journal will focus on the impact of management

\section{Dovepress}

programs and new therapeutic agents and protocols on patient perspectives such as quality of life, adherence and satisfaction. The manuscript management system is completely online and includes a very quick and fair peer-review system, which is all easy to use. Visit http://www.dovepress.com/testimonials.php to read real quotes from published authors 